\title{
Treatment with broad-spectrum antibiotics upregulates Sglt1 and induces small intestinal villous hyperplasia in mice
}

\author{
Takayuki Ota, ${ }^{1}$ Takeshi Ishikawa, ${ }^{1,2, *}$ Tomoki Sakakida, ${ }^{1}$ Yuki Endo, ${ }^{1}$ Shinya Matsumura, ${ }^{1}$ Juichirou Yoshida, ${ }^{1}$ \\ Yasuko Hirai, ${ }^{1}$ Katsura Mizushima, ${ }^{1}$ Kaname Oka, ${ }^{1}$ Toshifumi Doi, ${ }^{1}$ Tetsuya Okayama, ${ }^{1}$ Ken Inoue, ${ }^{1}$ \\ Kazuhiro Kamada, ${ }^{1}$ Kazuhiko Uchiyama, ${ }^{1}$ Tomohisa Takagi, ${ }^{1}$ Hideyuki Konishi, ${ }^{1}$ Yuji Naito, ${ }^{1}$ and Yoshito Itoh $^{1}$
}

${ }^{1}$ Molecular Gastroenterology and Hepatology and ${ }^{2}$ Outpatient Oncology Unit, University Hospital, Kyoto Prefectural University of Medicine, 465 Kajii-cho, Hirokoji Kawaramachi, Kamigyo-ku, Kyoto 602-8566, Japan

\begin{abstract}
Although extensive evidence indicates that the gut microbiota plays a crucial role in regulating glucose homeostasis, the exact regulatory mechanism remains unclear. This study aimed to investigate the effect of broad-spectrum antibiotics on the expression of glucose transporters, histomorphology of the small intestine, and glucose metabolism in mice. C57BL/6 mice were administered drinking water with or without a broad-spectrum antibiotic combination for 4 weeks. Thereafter, an oral glucose tolerance test was performed. Body weight, small intestine histopathology, mRNA levels of glucose transporters (SGLT1 and GLUT2) and intestinal transcription factors (CDX1 and CDX2) were evaluated. SGLT1 and CDX1 were upregulated in the small intestine upon antibiotic administration compared with that in the control group. The height and surface area of the jejunal villi were significantly higher upon antibiotic administration than in the control group. Fasting glucose levels were significantly higher upon antibiotic administration than in the control group. The present results indicate that treatment with broad-spectrum antibiotics upregulates SGLT1 and CDX1 and induces hyperplasia in the small intestine, thus increasing fasting blood glucose levels. Our results further the current understanding of the effects of broad-spectrum antibiotics on the gut microbiota and glucose homeostasis that may have future clinical implications.
\end{abstract}

Key Words: antibiotics, gut microbiota, SGLT1, intestinal hyperplasia, glucose tolerance

ntibiotics are widely used for treating and preventing pathogenic infections; however, they can cause dysbiosis and affect human health. Antibiotic-induced dysbiosis is a risk factor for obesity, metabolic disorders, and diabetes mellitus (DM). (1,2) Treatment with a combination of broad-spectrum antibiotics in early life accelerates the onset of DM in adulthood in mice and humans. ${ }^{(3-6)}$ Changes in the abundance of Akkermansia muciniphilia, Lactobacillus spp., Clostridium spp., and Bacteroides spp. have been reported in patients with diabetes and obesity. ${ }^{(7-9)}$ Moreover, probiotics potentially alter glucose homeostasis in mice and humans. ${ }^{(10,11)}$ Thus, the intestinal microbiota plays an important role in regulating glucose homeostasis. Despite advancements in the understanding of the basis of host-microbiome symbiosis, the exact mechanism through which the gut microbiota potentially regulates host glucose metabolism remains unclear.

In the small intestine, glucose absorption is mediated by sodium-dependent glucose transporter 1 (SGLT1) and facilitative glucose transporter 2 (GLUT2), which are associated with incretin responses. SGLT1 is apically expressed and transports glucose from the intestinal lumen to the cytosol of enterocytes, whereas GLUT2 is basolaterally expressed and transports glucose from the cytosol of enterocytes to the blood. ${ }^{(12-14)}$ Intestinal SGLT1 is reportedly upregulated in diabetes and this glucose transporter has been speculated as a novel therapeutic target in diabetes. ${ }^{(15-18)}$ Although intestinal glucose absorption regulates glucose homeostasis and the gut microbiota is an important regulator of glucose homeostasis, ${ }^{(19)}$ the effects of antibiotic-induced dysbiosis on intestinal glucose transporters are unclear. Therefore, this study aimed to investigate the effect of broad-spectrum antibiotics on expression of the glucose transporters (SGLT1 and GLUT2), histomorphology of the small intestine, and glucose metabolism in mice.

\section{Materials and Methods}

Mice. A total of 24 seven-week-old C57BL/6 mice (Shimizu Laboratory Supplies, Kyoto, Japan) were acclimated for one week in the animal facility of the Division of Animal Experiment, Kyoto Prefectural University of Medicine in accordance with the guidelines for Institutional Animal Care, in plastic cages ( $n=5$ or 6 mice per cage) with ad libitum access to drinking water and a basal diet under controlled conditions of humidity, light (12/12-h light/dark) and temperature. All animal experiments and experiments were approved by the Experimental Animal Research Committee of the Kyoto Prefecture University of Medicine.

Segregation of mice and extraction of mouse tissue. After a week of acclimatization, the mice were segregated into two body-weight-matched groups $(n=12)$. Antibiotic-treated mice were treated with a combination of broad-spectrum antibiotics $(1 \mathrm{~g} / \mathrm{L}$ ampicillin, $500 \mathrm{mg} / \mathrm{L}$ vancomycin, and $1 \mathrm{~g} / \mathrm{L}$ neomycin sulfate) in drinking water. ${ }^{(20)}$ Food intake and body weights were recorded every day. Four weeks after antibiotic administration, all mice were euthanized via cervical dislocation. Cecum and epididymal adipose tissue were extracted and weighed and 2-cm jejunum segments approximately $4 \mathrm{~cm}$ distal to the ligament of Treitz were harvested. Jejunum tissue was divided into two parts, of which one was snap-frozen in liquid nitrogen and stored at $-80^{\circ} \mathrm{C}$ until use, while the other part was fixed in $10 \%$ formaldehyde for $24 \mathrm{~h}$, dehydrated, and embedded in paraffin.

*To whom correspondence should be addressed. E-mail: iskw-t@koto.kpu-m.ac.jp 
Oral glucose tolerance test (OGTT). Four weeks after antibiotic administration, OGTT was performed among all mice. After $16 \mathrm{~h}$ of fasting with ad libitum access to water, mice were orally administered $40 \%$ glucose with a feeding gavage (at $2 \mathrm{~g} / \mathrm{kg}$ body weight), using a stainless-steel gavage needle. Blood samples were obtained from the tail vein before and after 30,60, 90 , and $120 \mathrm{~min}$ of glucose administration. Blood glucose was measured using a glucometer. Area under the curve (AUC) values for plasma glucose were determined.

Quantitative reverse transcription-PCR analysis. Sglt1, Glut2, $C d x 1$, and $C d x 2$ expression levels were quantified via real-time PCR. Total RNA was isolated from homogenized mouse tissue, using the acid guanidinium phenol chloroform method with Isogen (Nippon Gene Co. Ltd., Tokyo, Japan) and stored at $-80^{\circ} \mathrm{C}$ until use. Thereafter, $1 \mu \mathrm{g}$ of extracted RNA was reverse-transcribed into first-strand cDNA, using the High Capacity cDNA Reverse Transcription kit (Applied Biosystems, Foster City, CA) in accordance with the manufacturer's instructions. Real-time PCR for Sglt1, Glut2, $C d x 1, C d x 2$, and Actb was carried out using the 7300 Real-time PCR system (Applied Biosystems) using the DNA-binding dye SYBR ${ }^{\circledR}$ Green to detect the PCR products. The following primers were used: Sglt1, sense 5'-CCTCTCGGCCAAGAACATGT-3' and antisense 5'-TTC AGATAGCCACACAGGGTACA-3'; Glut2, sense 5'-TGGAAG GATCAAAGCAATGTTG-3' and antisense 5'-CATCAAGAG GGCTCCAGTCAA-3'; $C d x 1$, sense 5'-CGCCTAGAGCTGGAA AAGGA-3' and antisense 5'-TTAGCAGCCAGCTCGGACTT-3'; $C d x 2$, sense 5'-CGAGCCCTTGAGTCCTGTGA-3' and antisense 5'-AACCCCAGGGACAGAACCA-3'; Actb, sense 5'-TATCCA CCTTCCAGCAGATGT-3' and antisense 5'-AGCTCAGTAACA GTCCGCCTA-3'.

Histopathological analysis. Small intestinal specimens were gently rinsed with normal saline, fixed in formalin solution, and embedded in paraffin. Sections were deparaffinized, hydrated, and stained with hematoxylin and eosin. Histopathological findings were evaluated using a microscope (BX50; Olympus, Tokyo, Japan) in a blinded manner. Villar height and width and crypt depth were measured for six mice and segments in each group. Villar surface area was determined as follows: $\pi \times$ villar length $\times$ villar width. ${ }^{(21)}$

Microbiome analysis. Microbiome analysis was performed according to the previous reports. ${ }^{(22,23)}$ Briefly, the QIAamp Fast DNA Stool Mini Kit (Qiagen, Valencia, CA) was used to extract genomic DNA from the collected fecal pellets. Microbiome analyses by $16 \mathrm{~S}$ rRNA gene sequencing were conducted by Takara Bio Inc. DNA specimen from feces was amplified using a 16S (V3-V4) metagenomic library construction kit for NGS (Takara Bio Inc., Kusatsu, Japan) with primer pairs 341F (5'TCGTCGGCAGCGTCAGATGTGTATAAGAGACAGCCTACG GGNGGCWGCAG-3') and 806R (5'-GTCTCGTGGGCTCGG AGATGTGTATAAGAGACAGGGACTACHVGGGTWTCTAAT$\left.3^{\prime}\right)$ corresponding to the $\mathrm{V} 3-\mathrm{V} 4$ region of the $16 \mathrm{~S}$ rRNA gene. The amplicons were purified and prepared for the sequencing library by using AMPure XP beads (Beckman Coulter, Brea, CA). The purified amplicon library was sequenced on an Illumina Miseq platform (Illumina Inc., San Diego, CA) at the Biomedical Center at Takara Bio. The processing of sequence data, including chimera check, operational taxonomic unit (OTU) definition, and taxonomy assignment, was performed using QIIME 2, ${ }^{(24)}$ DATA2, ${ }^{(25)}$ and VSEARCH. ${ }^{(26)}$

Statistical analysis. Data are expressed as mean $\pm \mathrm{SE}$ of the mean values. Differences between the antibiotic-treated and control groups were assessed via the Student's $t$ test. Differences were considered significant at a two-sided $p$ value $<0.05$. All statistical analyses were performed using JMP 13 software (SAS Institute, Inc., Cary, NC).

\section{Results}

Effect of antibiotics on body weight and epididymal adipose tissue. On examining food intake per period, food intake decreased in the antibiotic-treated group compared with that in the control group in the initial period (days 1-7) $(p=$ 0.0002); however, thereafter, no significant difference in food intake was observed between the two groups (Supplemental Fig. $1 *)$. Body weight did not significantly differ between the two groups at the end of the experiment (Fig. 1A). However, the cecum weight was significantly greater in the antibiotic-treated group than in the control group $(p<0.0001)$, and consequently, the average body weight excluding the cecum weight was significantly lower in the in antibiotic-treated group than in the control group ( $p=0.023$ ) (Fig. 1B-D). Epididymal adipose weight was significantly lower in the antibiotic-treated group than in the control group ( $p=0.035)$ (Fig. 1E).

Effect of antibiotics on the expression of intestinal glucose transporters. SGLT1 expression levels in jejunal epithelial cells were significantly higher in the antibiotic-treated group than in the control group ( $p=0.009$, Fig. $2 \mathrm{~A})$; however, GLUT2 expression levels did not differ significantly between the two groups (Fig. 2B). Immunostaining showed that SGLT1 expressed mainly on the apical pole of epithelial cells continuously in antibiotics-treated mice (Fig. 2C).

Effect of antibiotics on the histopathological features of the intestinal mucosa. The jejunal villar height and surface area were significantly greater in the antibiotic-treated group than in the control group $(p<0.001)$, while crypt depth and villar width did not significantly differ. Regular villar morphology was retained in the antibiotic-treated group (Fig. 3A and B).

Furthermore, on analyzing CDX1/2 expression levels, both intestinal transcription factors, CDX1 mRNA levels were significantly higher in the antibiotic-treated group than in the control group ( $p=0.028$, Fig. 3C-1), whereas CDX2 mRNA levels did not significantly differ between the two groups (Fig. 3C-2).

OGTT. Fasting glucose levels were significantly higher in the antibiotic-treated group than in the control group $(p=0.043)$ (Fig. 4A). However, the OGTT revealed no significant differences in plasma glucose levels after a glucose load $(30,60,90$, and $120 \mathrm{~min}$ ) between the two groups (Fig. 4A). Furthermore, the average AUC for plasma glucose did not differ between the two groups (Fig. 4B).

Gut microbiota diversity and composition. The effects of a broad-spectrum antibiotic combination on the intestinal microbiota were investigated. As for the diversity of gut microbiota using different alpha-diversity indices (the Shannon and the Chao 1 index), both the values in antibiotic-treated group were significantly higher than the control group $(p=0.0049$ and $p=0.0062$, respectively, Supplemental Fig. 2A*). A PCoA analysis using the weighted UniFrac suggested that overall community composition of the microbiota from the antibiotictreated group was very different from the control group (Supplemental Fig. 2B*). Subsequently, the differences in the gut microbial structure in two groups were taxonomically evaluated at the phylum level. Compared to the control, antibiotic-treated group had a lower abundance of the phyla Bacteroidetes and Verrucomicrobia, and higher abundance of the phyla Proteobacteria (Fig. 5A). The Firmicutes/Bacteroidetes ratio was higher in the antibiotic-treated group than in the control group, though it did not reach statistical significance ( $p=0.11$, Fig. 5B).

\section{Discussion}

The gut microbiota has recently been recognized as an important regulator of glucose homeostasis. Previous reports indicate that there is a cause-effect relationship between the gut microbiota and insulin sensitivity, and several mechanisms 

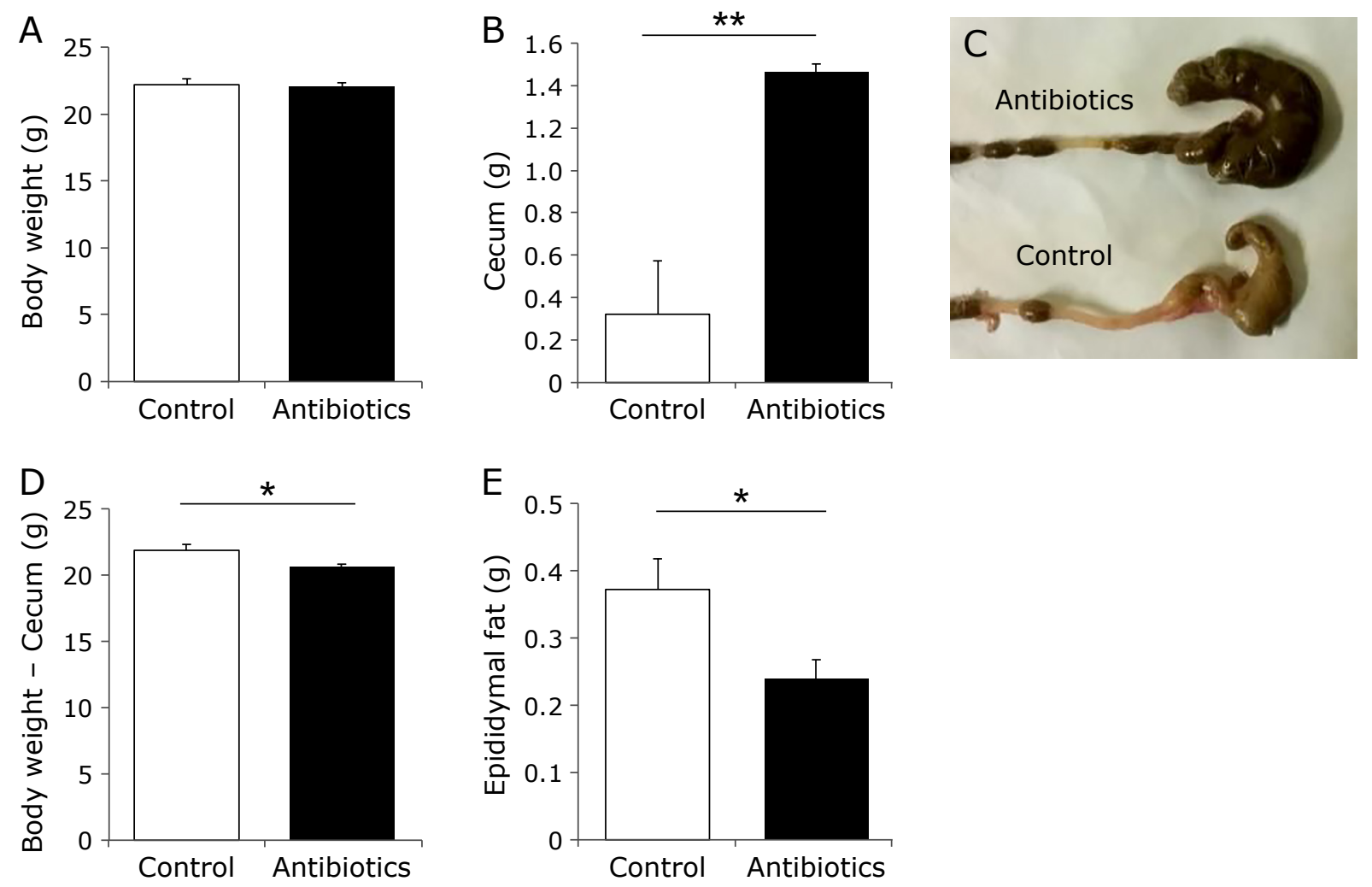

Fig. 1. Body, cecum and epididymal fat weight. (A) Body weight did not differ between groups. (B, C) Cecum weight was significantly higher in the antibiotic-treated group than in the control group. (D) Hence, the body weight, excluding the cecum weight, was significantly lower in the antibiotic-treated group. (E) The weight of epididymal fat was significantly lower in the antibiotic-treated group than in the control group. Values are expressed as mean with SE of six mice in each group. ${ }^{*} p<0.05, * * p<0.001$.
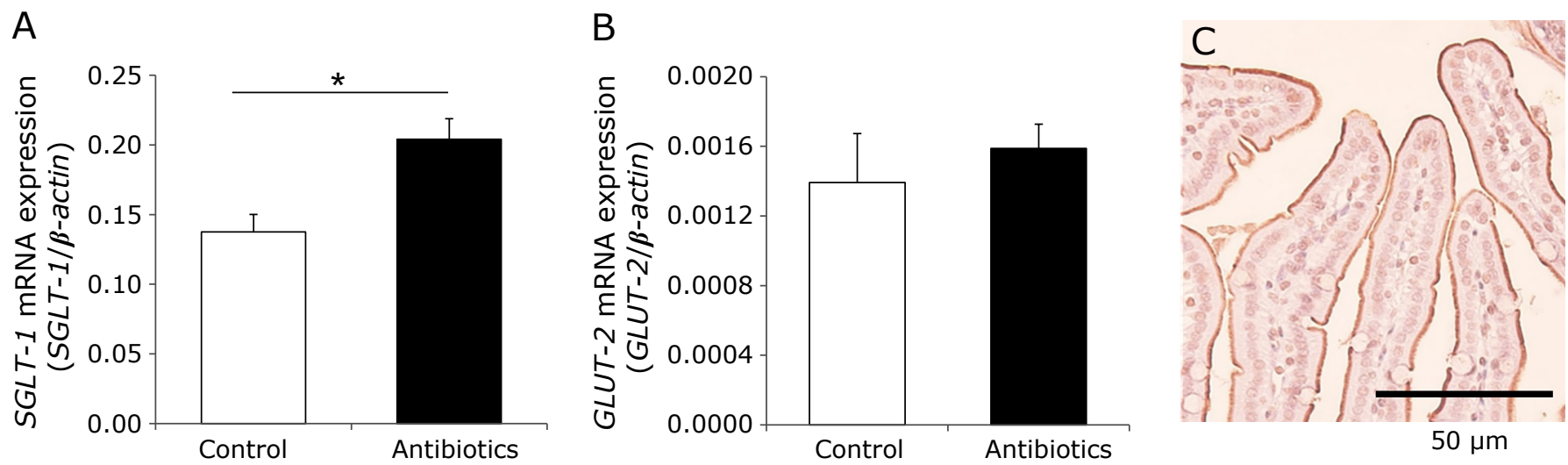

Fig. 2. Sglt1 and Glut2 mRNA expression levels. (A) Sglt1 mRNA levels were significantly higher in the antibiotic-treated group than in the control group. (B) However, no differences in Glut2 mRNA levels were observed in both groups. (C) SGLT1 was expressed mainly in the apical pole of epithelial cells in mice treated with antibiotics. Values are expressed as mean with SE of six mice in each group. * $p<0.05$.

contributing to explain these associations are assumed. Lipopolysaccharide (LPS) from gut microbiota can induce a chronic subclinical inflammation, leading to insulin resistance, and short-chain fatty acids (SCFA) also have an essential role on the regulation of insulin sensitivity. ${ }^{(27,28)}$ Other mechanisms including bile acids and branched-chain amino acid (BCAA), ${ }^{(29,30)}$ all of whose composition are changed by gut dysbiosis, were also proposed, however, few studies have focused on the intestinal glucose transporters in the relationship between the gut microbiota and glucose homeostasis. The present results show that long-term use of broad-spectrum antibiotics upregulates jejunal
SGLT1. Furthermore, the jejunal villar height and surface area and CDX1 expression levels in the antibiotic-treated mice were significantly higher than those in the control group. To our best knowledge, our study is the first to show that long-term use of broad-spectrum antibiotics potentially affects the expression of glucose transporters in the small intestine.

SGLT1 is upregulated by DM, ${ }^{(16)}$ tacrolimus, ${ }^{(31)} \alpha-$ ketoglutarate, ${ }^{(32)}$ and vitamin $\mathrm{D}$ and downregulated by a lowcarbohydrate high-protein diet or duodenojejunal bypass. ${ }^{(33-35)}$ Numerous factors alter SGLT1 expression levels in the small intestine; however, the mechanisms regulating SGLT1 remain 
A Control
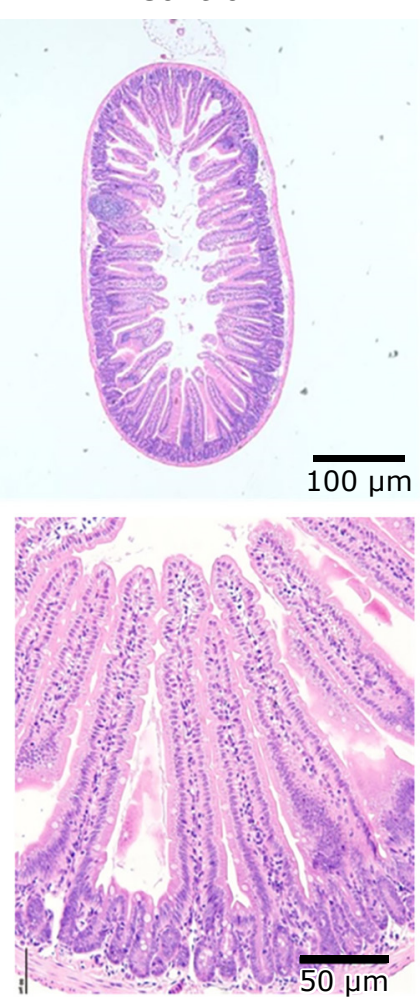

C

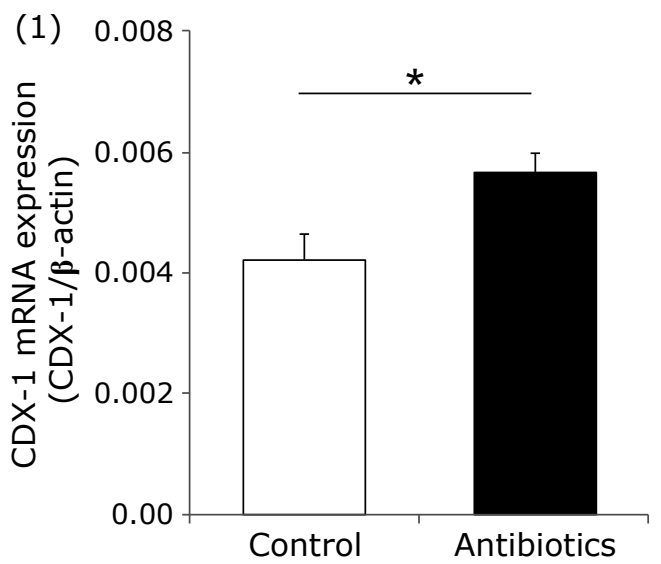

Antibiotics

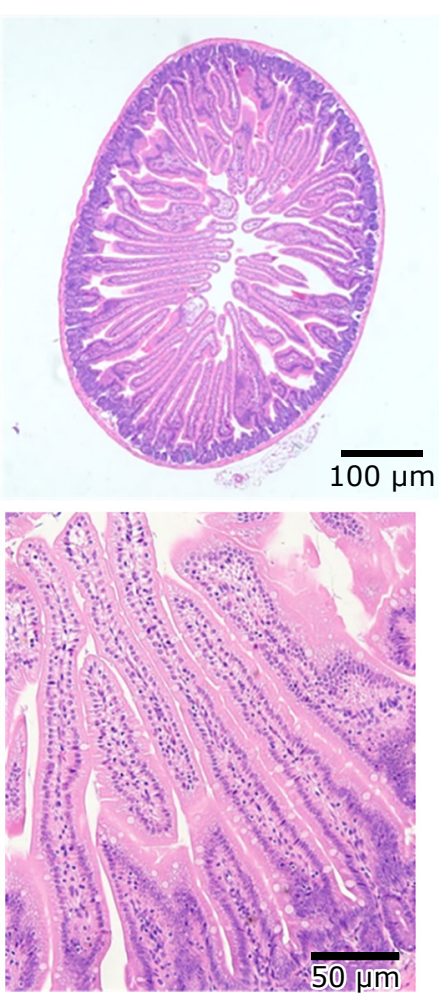

B
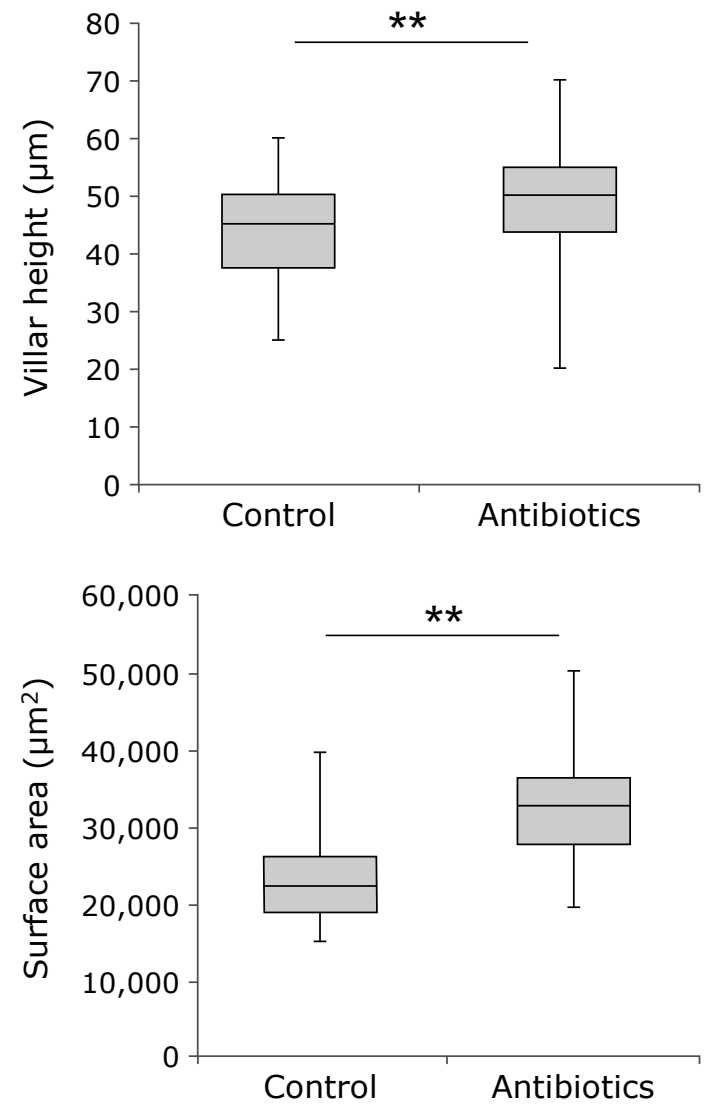

Fig. 3. Intestinal histology, and $C d x 1$ and $C d x 2$ mRNA expression levels. (A) Micrographs of representative intestinal sections from the two groups (hematoxylin and eosin staining, $\times 40$ magnification). (B) Villar height and villar surface area were significantly greater in the antibiotic-treated group than in the control group. (C) Cdx1 mRNA levels were significantly higher in the antibiotic-treated group. (D) However, no difference in Cdx2 mRNA levels were observed in both groups. Values are expressed as median with 25th and 75th percentiles (B) or mean with SE (C) of six mice in each group. ${ }^{*} p<0.05,{ }^{* *} p<0.001$.

unclear. This study shows that treatment with broad-spectrum antibiotics upregulates jejunal SGLT1, which is reportedly elevated in germ-free (GF) mice. ${ }^{(36)}$ A major role of the gut microbiota is in energy extraction from undigested polysaccharides via the release of SCFAs. This energy extraction by the gut microbiota is lost in GF mice. Antibiotics-treatment also reduced the levels of microbial-derived SCFAs. ${ }^{(37)}$ It is reasonable to assume that jejunal SGLT1 upregulation was induced to increase glucose absorption to compensate for reduced energy supply. Although the exact mechanisms under- lying SGLT1 upregulation in this study remains unclear, it is possible that gut microbiota is directly or indirectly involved. Microbiome analysis revealed that the Firmicutes/Bacteroidetes ratio in antibiotic-treated mice was elevated, which is also observed in obese/insulin resistant individuals. ${ }^{(38)}$ In line with our results, Adachi et al. ${ }^{(39)}$ recently demonstrated that patients with type $2 \mathrm{DM}$ had more Lactobacillales, belonging to the phylum Firmicutes, in their feces and less Bacteroides spp. compared to healthy individuals. Furthermore, it has been reported that SGLT1 expression in the small intestine is increased in obese 

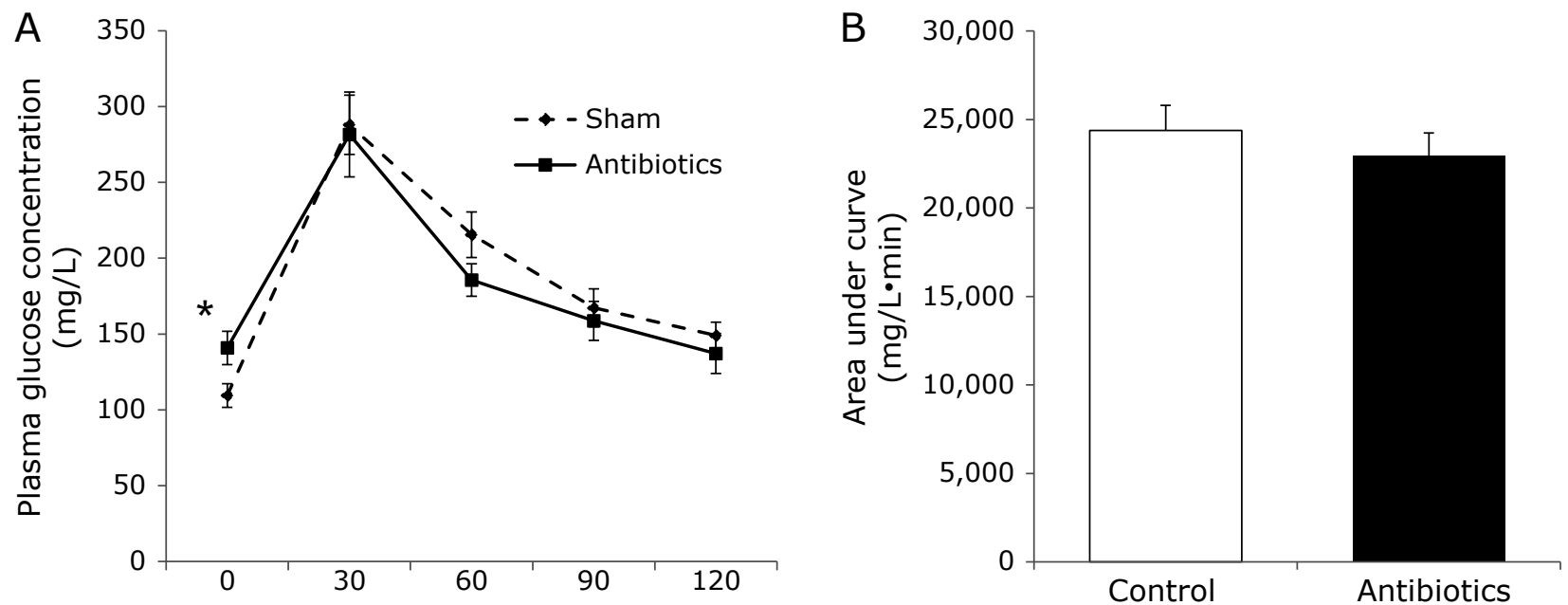

Fig. 4. Effect of antibiotics on plasma glucose levels. (A) Fasting glucose levels were significantly higher in the antibiotic-treated group than in the control group. However, no significant differences were observed at 30,60, 90, and $120 \mathrm{~min}$. (B) No significant differences were observed in the area under curve values for plasma glucose. Values are expressed as mean with SE of six mice in each group. * $p<0.05$.

A

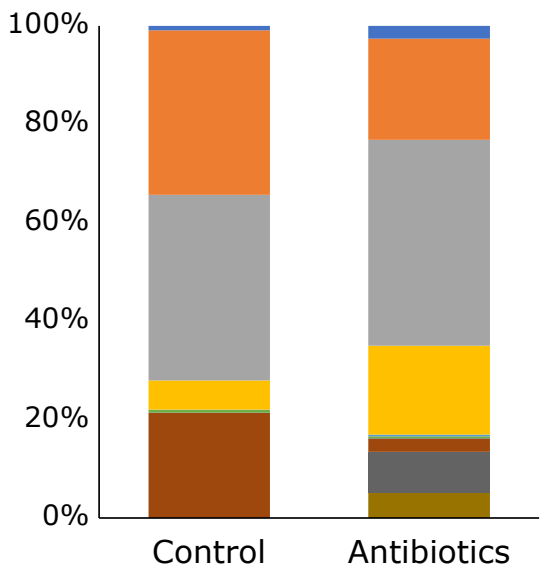

B Firmicutes/Bacteroidetes ratio

- Actinobacteria

Bacteroidetes

- Firmicutes

- Proteobacteria

- Deferribacteres

- TM7

- Tenericutes

- Verrucomicrobia

- Cyanobacteria

- Others

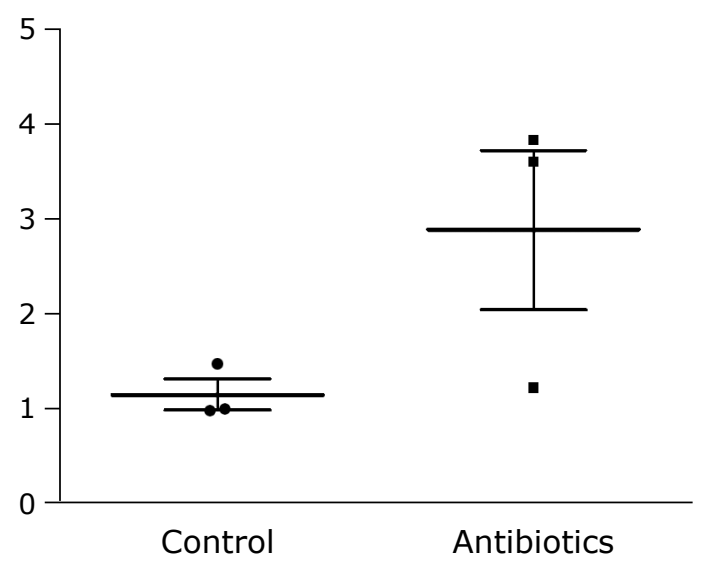

Fig. 5. Effect of antibiotics on gut microbiota. (A) The microbial composition at the phylum level. (B) Firmicutes/Bacteroidetes ratio. The Firmicutes/Bacteroides phylum ratio tended to be higher in the antibiotic-treated group than in the control group. Values are expressed as mean with SE of three mice in each group.

individuals. ${ }^{(40)}$ Altered microbiota composition induced by broad-spectrum antibiotics treatment, including changes in the Firmicutes/Bacteroidetes ratio, may affect SGLT1 expression. The relationship between intestinal glucose transporter and gut microbiota and the mechanisms by which the gut microbiota can alter the intestinal SGLT1 expression need to be clarified by future studies.

Herein, fasting glucose levels were significantly higher in the antibiotic-treated group; however, the OGTT revealed no difference in glucose levels after a glucose load between the two groups. In the small intestine, SGLT1 is expressed in both enterocytes and enteroendocrine cells. Enterocyte SGLT1 is primarily responsible for glucose absorption, while glucose uptake by SGLT1 in enteroendocrine cells stimulates the release of incretins including glucagon-like peptide-1 (GLP-1). ${ }^{(41)}$ Glucose-induced incretin, GLP-1 and glucose-dependent insulinotropic peptide regulate blood glucose levels via several mechanisms, including amplification of glucose-induced insulin secretion. These findings suggest that SGLT1 upregulation potentially promotes incretin secretion after a glucose load. Differences in glucose levels in fasting and post-glucose loading states between the two groups may be explained in part on the basis of the reactive secretion of incretin after a glucose load.

Furthermore, we found that jejunal villar height and surface area were significantly greater in the antibiotic-treated group than in the control group. Moreover, CDX1 expression levels were higher in the antibiotic-treated group than in the control group. Caudal-related homeobox genes $c d x 1$ and $c d x 2$ are intestinal transcription factors playing critical roles in intestinal cell proliferation and differentiation. ${ }^{(42)} \mathrm{CDX}$ expression in the small intestine is reportedly induced during starvation. ${ }^{(43,44)}$ Herein, fasting glucose levels and epididymal adipose weight were significantly lower in the antibiotics group than in the control group. Therefore, CDX1 expression and consequent jejunal epithelial hyperplasia might be induced in response to starvationlike conditions resulting from the reduction in the energy supply associated with antibiotic-induced dysbiosis. However, CDX1 expression and hyperplasia have been reported in the small intestine in a rat model of DM. ${ }^{(42)}$ Since various factors are potentially involved in regulating $\mathrm{CDX}$ expression, and the microbiota may directly affect CDX expression, further studies are needed to elucidate the mechanism underlying antibiotic- 
induced CDX expression.

In conclusion, our study showed that treatment with broadspectrum antibiotics potentially upregulates SGT1 and CDX1 and induces hyperplasia in the jejunum, thereby increasing fasting blood glucose levels. Although the mechanism underlying the regulation of glucose homeostasis by the gut microbiota appears complex, our results indicate that SGLT1 is partially involved in glucose regulation via the gut microbiota. These findings further the current understanding of the effect of broad-spectrum antibiotics on the gut microbiota and glucose homeostasis.

\section{Author Contributions}

The conception and design of the work: TO and TI; Acquisition of data: TO, TS, YE, SM, JY, YH, KM, and KO; Analysis and interpretation of data: TO, TI, TD, TO, KI, KK, KU, TT, HK, YN, and YI; Drafting the work: TO and TI. All authors approved the final version of this manuscript to be published.

\section{References}

1 Turnbaugh PJ, Backhed F, Fulton L, Gordon JI. Diet-induced obesity is linked to marked but reversible alterations in the mouse distal gut microbiome. Cell Host Microbe 2008; 3: 213-223.

2 Caricilli AM, Saad MJ. The role of gut microbiota on insulin resistance. Nutrients 2013; 5: 829-851.

3 Candon S, Perez-Arroyo A, Marquet C, et al. Antibiotics in early life alter the gut microbiome and increase disease incidence in a spontaneous mouse model of autoimmune insulin-dependent diabetes. PLoS One 2015; 10: e0125448.

4 Brown K, Godovannyi A, Ma C, et al. Prolonged antibiotic treatment induces a diabetogenic intestinal microbiome that accelerates diabetes in NOD mice. ISME J 2016; 10: 321-332.

5 Boursi B, Mamtani R, Haynes K, Yang YX. The effect of past antibiotic exposure on diabetes risk. Eur J Endocrinol 2015; 172: 639-648.

6 Mikkelsen KH, Knop FK, Frost M, Hallas J, Pottegard A. Use of antibiotics and risk of type 2 diabetes: a population-based case-control study. J Clin Endocrinol Metab 2015; 100: 3633-3640.

7 Sanz Y, Olivares M, Moya-Pérez Á, Agostoni C. Understanding the role of gut microbiome in metabolic disease risk. Pediatr Res 2015; 77: 236-244.

8 Qin J, Li Y, Cai Z, et al. A metagenome-wide association study of gut microbiota in type 2 diabetes. Nature 2012; 490: 55-60.

9 Karlsson FH, Tremaroli V, Nookaew I, et al. Gut metagenome in European women with normal, impaired and diabetic glucose control. Nature 2013; 498: 99-103.

10 Balakumar M, Prabhu D, Sathishkumar C, et al. Improvement in glucose tolerance and insulin sensitivity by probiotic strains of Indian gut origin in high-fat diet-fed C57BL/6J mice. Eur J Nutr 2018; 57: 279-295.

11 Ejtahed HS, Mohtadi-Nia J, Homayouni-Rad A, Niafar M, Asghari-Jafarabadi M, Mofid V. Probiotic yogurt improves antioxidant status in type 2 diabetic patients. Nutrition 2012; 28: 539-543.

12 Wright EM, Loo DD, Hirayama BA. Biology of human sodium glucose transporters. Physiol Rev 2011; 91: 733-794.

13 Yoshikawa $\mathrm{T}$, Inoue $\mathrm{R}$, Matsumoto $\mathrm{M}$, Yajima $\mathrm{T}$, Ushida $\mathrm{K}$, Iwanaga $\mathrm{T}$. Comparative expression of hexose transporters (SGLT1, GLUT1, GLUT2 and GLUT5) throughout the mouse gastrointestinal tract. Histochem Cell Biol 2011; 135: 183-194.

14 Wolffram S, Block M, Ader P. Quercetin-3-glucoside is transported by the glucose carrier SGLT1 across the brush border membrane of rat small intestine. J Nutr 2002; 132: 630-635.

15 Ogata H, Seino Y, Harada N, et al. KATP channel as well as SGLT1 participates in GIP secretion in the diabetic state. J Endocrinol 2014; 222: 191-200.

16 Gorboulev V, Schürmann A, Vallon V, et al. $\mathrm{Na}^{+}$-D-glucose cotransporter SGLT1 is pivotal for intestinal glucose absorption and glucose-dependent incretin secretion. Diabetes 2012; 61: 187-196.

17 Dyer J, Wood IS, Palejwala A, Ellis A, Shirazi-Beechey SP. Expression of monosaccharide transporters in intestine of diabetic humans. Am J Physiol

\section{Acknowledgments}

We thank our colleagues in the department of Molecular Gastroenterology and Hepatology for helpful discussion and valuable assistance. This work was supported by JSPS KAKENHI Grant Number 20K07787 and 17K09314.

\section{Abbreviations}

$\begin{array}{ll}\text { AUC } & \text { area under the curve } \\ \text { DM } & \text { diabetes mellitus } \\ \text { GF } & \text { germ-free } \\ \text { GLP } & \text { glucagon-like peptide } \\ \text { GLUT } & \text { glucose transporter } \\ \text { OGTT } & \text { oral glucose tolerance test } \\ \text { SGLT } & \text { sodium-dependent glucose transporter }\end{array}$

\section{Conflict of Interest}

No potential conflicts of interest were disclosed.

Gastrointest Liver Physiol 2002; 282: G241-G248.

18 Song P, Onishi A, Koepsell H, Vallon V. Sodium glucose cotransporter SGLT1 as a therapeutic target in diabetes mellitus. Expert Opin Ther Targets 2016; 20: 1109-1125.

19 Shirazi-Beechey SP, Moran AW, Batchelor DJ, Daly K, Al-Rammahi M. Glucose sensing and signalling; regulation of intestinal glucose transport. Proc Nutr Soc 2011; 70: 185-193.

$20 \mathrm{Xu} \mathrm{C}$, Ruan B, Jiang Y, et al. Antibiotics-induced gut microbiota dysbiosis promotes tumor initiation via affecting APC-Th1 development in mice. Biochem Biophys Res Commun 2017; 488: 418-424.

21 Kisielinski K, Willis S, Prescher A, Klosterhalfen B, Schumpelick V. A simple new method to calculate small intestine absorptive surface in the rat. Clin Exp Med 2002; 2: 131-135.

22 Higashimura Y, Baba Y, Inoue R, et al. Agaro-oligosaccharides regulate gut microbiota and adipose tissue accumulation in mice. J Nutr Sci Vitaminol (Tokyo) 2017; 63: 269-276.

23 Fujita S, Baba Y, Nakashima Y, et al. Administration of Enterococcus faecium HS-08 increases intestinal acetate and induces immunoglobulin A secretion in mice. Can J Microbiol 2020; 66: 576-585.

24 Bolyen E, Rideout JR, Dillon MR, et al. Reproducible, interactive, scalable and extensible microbiome data science using QIIME 2. Nat Biotechnol 2019; 37: $852-857$.

25 Callahan BJ, McMurdie PJ, Rosen MJ, Han AW, Johnson AJ, Holmes SP DADA2: high-resolution sample inference from Illumina amplicon data. Nat Methods 2016; 13: 581-583.

26 Edgar RC. Search and clustering orders of magnitude faster than BLAST. Bioinformatics 2010; 26: 2460-2461.

27 Canfora EE, Jocken JW, Blaak EE. Short-chain fatty acids in control of body weight and insulin sensitivity. Nat Rev Endocrinol 2015; 11: 577-591.

28 Saad MJ, Santos A, Prada PO. Linking gut microbiota and inflammation to obesity and insulin resistance. Physiology (Bethesda) 2016; 31: 283-293.

29 Thomas C, Gioiello A, Noriega L, et al. TGR5-mediated bile acid sensing controls glucose homeostasis. Cell Metab 2009; 10: 167-177.

30 Tremblay F, Krebs M, Dombrowski L, et al. Overactivation of S6 kinase 1 as a cause of human insulin resistance during increased amino acid availability. Diabetes 2005; 54: 2674-2684.

31 Li Z, Sun F, Zhang Y, et al. Tacrolimus induces insulin resistance and increases the glucose absorption in the jejunum: a potential mechanism of the diabetogenic effects. PLoS One 2015; 10: e0143405.

32 He L, Zhou X, Huang N, et al. Administration of alpha-ketoglutarate improves epithelial restitution under stress injury in early-weaning piglets. Oncotarget 2017; 8: 91965-91978.

33 Rexhepaj R, Alesutan I, Gu S, et al. SGK1-dependent stimulation of intestinal SGLT1 activity by vitamin D. Pflugers Arch 2011; 462: 489-494.

34 Higashida K, Terada S, Li X, et al. Low-carbohydrate high-protein diet 
diminishes the insulin response to glucose load via suppression of SGLT-1 in mice. Biosci Biotechnol Biochem 2019; 83: 365-371.

35 Yan S, Sun F, Li Z, et al. Reduction of intestinal electrogenic glucose absorption after duodenojejunal bypass in a mouse model. Obes Surg 2013; 23: 1361-1369.

36 Swartz TD, Duca FA, de Wouters T, Sakar Y, Covasa M. Up-regulation of intestinal type 1 taste receptor 3 and sodium glucose luminal transporter-1 expression and increased sucrose intake in mice lacking gut microbiota. $\mathrm{Br} J$ Nutr 2012; 107: 621-630.

37 Guinan J, Wang S, Hazbun TR, Yadav H, Thangamani S. Antibiotic-induced decreases in the levels of microbial-derived short-chain fatty acids correlate with increased gastrointestinal colonization of Candida albicans. Sci Rep 2019; 9: 8872 .

38 Magne F, Gotteland M, Gauthier L, et al. The Firmicutes/Bacteroidetes ratio: a relevant marker of gut dysbiosis in obese patients? Nutrients 2020; 12: 1474.

39 Adachi K, Sugiyama T, Yamaguchi Y, et al. Gut microbiota disorders cause type 2 diabetes mellitus and homeostatic disturbances in gut-related metabolism in Japanese subjects. J Clin Biochem Nutr 2019; 64: 231-238.

40 Nguyen NQ, Debreceni TL, Bambrick JE, et al. Accelerated intestinal glucose absorption in morbidly obese humans: relationship to glucose transporters, incretin hormones, and glycemia. J Clin Endocrinol Metab 2015; 100: $968-976$.

41 Kuhre RE, Frost CR, Svendsen B, Holst JJ. Molecular mechanisms of glucose-stimulated GLP-1 secretion from perfused rat small intestine. Diabetes 2015; 64: 370-382.

42 Adachi T, Mori C, Sakurai K, Shihara N, Tsuda K, Yasuda K. Morphological changes and increased sucrase and isomaltase activity in small intestines of insulin-deficient and type 2 diabetic rats. Endocr J 2003; 50: 271-279.

43 Matsuo T, Odaka H, Ikeda H. Effect of an intestinal disaccharidase inhibitor (AO-128) on obesity and diabetes. Am J Clin Nutr 1992; 55 (1 Suppl): 314S$317 \mathrm{~S}$.

44 Teno S, Nakajima-Uto Y, Nagai K, et al. Treatment with alpha-glucosidase inhibitor for severe reactive hypoglycemia: a case report. Endocr $J$ 2000; 47: $437-442$.

This is an open access article distributed under the terms of the Creative Commons Attribution-NonCommercial-NoDerivatives License (http://creativecommons.org/licenses/by-nc-nd/4.0/). 